\title{
Maintaining precision calibrations with optimal copper spheres
}

\author{
Kenneth G. Foote \\ Institute of Marine Research, 5011 Bergen, Norway
}

(Received 22 January 1982; accepted for publication 30 November 1982)

\begin{abstract}
Effects of variations in quantities influencing the backscattering cross sections of solid elastic spheres are studied through a particular case. This is that of the 60-mm-diam copper sphere, which is being used worldwide to calibrate the $38-\mathbf{k H z}$ echo sounders instrumental in fisheries surveying. Derived dependences of the backscattering cross section include those characterizing the sphere, immersion medium, and equipment. Some of the dependences are sufficiently weak to permit their neglect in assigning the calibration value of the backscattering cross section, while others must be considered. In every case the dependences are calculable; given measurement of the calibration conditions, necessary corrections to the backscattering cross section can be determined and applied with maintenance of 0.1-dB accuracy. The wider use of copper spheres in acoustic calibrations, as in the radiation force method, is advocated. The practical use of optimal copper spheres in calibrating echo sounders and echo integrators is treated in detail in an appendix.
\end{abstract}

PACS numbers: 43.85.Vb, 43.20.Fn, 43.30.Dr

\section{INTRODUCTION}

Much of the history of acoustic scattering by solid elastic spheres has been theoretical. Occasional expressions of the intent ${ }^{1}$ or hope ${ }^{2}$ of identifying such bodies acoustically indicate an early envisaged application. More recent applications, which have been realized, are the use of solid spheres for calibration purposes. Dragonette et al. ${ }^{3}$ have used tungsten carbide spheres for comparing differential measurements over a wide frequency band. Machined copper spheres are currently being used to calibrate echo sounders and echo integrators on board fisheries research vessels, ${ }^{4}$ and have been proposed for more general use. ${ }^{5}$

It is this last-mentioned application which is addressed here. To show that solid copper spheres can be used for the precision calibration of hydroacoustic equipment, knowledge of the backscattering cross section or target strength must be demonstrated for those variable, but determinable conditions of likely encounter. To show that the same spheres are convenient, the several dependences must be shown to be weak.

The present study aims to fulfill both objects through an error-type analysis. In this, variations in the several scattering parameters are performed and the effects of these on the backscattering cross section are computed for a particular copper sphere, namely, the 60-mm-diam sphere used to calibrate the standard 38-kHz echo sounders employed in fisheries research. Derived graphical and algebraic expressions may provide practical guidelines for similar precision calibration applications.

\section{METHOD AND NUMERICAL EXAMPLE}

The phenomenon of sound scattering by solid elastic spheres is well understood. A comprehensive record of research is contained in this journal, which published Faran's seminal work in $1951 .{ }^{6}$ For present purposes it is sufficient to state the basic quantity of interest in sonar-type calibrations. This is the backscattering cross section $\sigma^{5}$

$$
\sigma=4 \pi \int_{0}^{\infty}|S F H|^{2} d \omega / \int_{0}^{\infty}|S H|^{2} d \omega,
$$

where $\omega$ denotes the frequency, $S$ the incident signal spectrum, $F$ the monochromatic backscattering amplitude of the sphere, and $H$ the receiver frequency response function.

Equation (1) serves as the common basis for all computations of the effects of parameter variations reported here. Thus the computations are not approximations, as they would be were the equation linearized; rather, they reflect the full interplay of factors depending on the sphere, immersion medium, and acoustic/signal processing equipment.

Throughout the computations, $S$ corresponds to a pulsed sinusoid and $H$ is identical to that of the Simrad EK$400 / 38$ echo sounder. ${ }^{5}$ The quantities of variation are contained in $F$. These are divided into two classes, termed intrinsic and extrinsic factors, as the quantity is associated with the sphere or is associated with the immersion medium or state of ensonification. The intrinsic factors comprise the sphere diameter $2 \mathrm{a}$, density $\rho_{1}$, and longitudinal and transverse sound speeds, $c_{1}$ and $c_{2}$, respectively. The extrinsic medium factors include the density $\rho$, sound speed $c$, and temperature $\tau$, and the extrinsic equipment parameters, the center frequency $f$ and duration $T$ of the transmit pulse. In performing variations with respect to $\tau$, a constant salinity of $35 \mathrm{ppt}$ is assumed and the temperature dependences of expansion coefficient ${ }^{7}$ and elasticity constants ${ }^{8}$ of the sphere material, copper, are ignored. The temperature dependence is incorporated in the medium density and sound speed through the dependences described in Refs. 9 and 10.

The variations are performed with respect to the following baseline or reference case: $2 a=60 \mathrm{~mm}, \rho_{1}=8.947 \mathrm{~g} /$ $\mathrm{cm}^{3}, c_{1}=4.760 \mathrm{~km} / \mathrm{s}, c_{2}=2.2885 \mathrm{~km} / \mathrm{s}, \rho=1.027 \mathrm{~g} / \mathrm{cm}^{3}$, $c=1.490 \mathrm{~km} / \mathrm{s}, \tau=10^{\circ} \mathrm{C}, f=38 \mathrm{kHz}$, and $T=1 \mathrm{~ms}$. With the exception of $\tau$, hence $\rho$ and $c$, these values define the nominal condition of calibration of the EK-400/38 echo sounder.

The particular diameter of $60 \mathrm{~mm}$ was determined by 


\begin{tabular}{|c|c|c|c|c|c|c|}
\hline Parameter type & Name & Symbol & Units & $\begin{array}{l}\text { Computational } \\
\text { range }\end{array}$ & $\begin{array}{l}\text { Ranges of } \\
\text { possible or } \\
\text { likely } \\
\text { encounter }\end{array}$ & References \\
\hline Intrinsic/sphere & Diameter & $2 a$ & $\mathbf{m m}$ & {$[59.9,60.1]$} & {$[59.94,60.06]$} & 4 \\
\hline Intrinsic/sphere & Density & $\rho_{1}$ & $\mathrm{~g} / \mathrm{cm}^{3}$ & {$[8.85,9.00]$} & {$[8.89,8.96]$} & 11,12 \\
\hline Intrinsic/sphere & $\begin{array}{l}\text { Longitudinal } \\
\text { sound speed }\end{array}$ & $c_{1}$ & $\mathrm{~km} / \mathrm{s}$ & {$[4.50,5.20]$} & {$[4.64,5.01]$} & 13,14 \\
\hline Intrinsic/sphere & $\begin{array}{l}\text { Transverse } \\
\text { sound speed }\end{array}$ & $c_{2}$ & $\mathrm{~km} / \mathrm{s}$ & {$[2.20,2.35]$} & {$[2.21,2.33]$} & 13,15 \\
\hline Extrinsic/medium & Density & $\rho$ & $\mathrm{g} / \mathrm{cm}^{3}$ & {$[0.99,1.04]$} & {$[0.996,1.028]$} & 9 \\
\hline Extrinsic/medium & Sound speed & $c$ & $\mathrm{~km} / \mathrm{s}$ & {$[1.40,1.55]$} & {$[1.40,1.55]$} & 10 \\
\hline Extrinsic/medium & Temperature & $\tau$ & ${ }^{\circ} \mathrm{C}$ & {$[0,30]$} & {$[0,30]$} & 4 \\
\hline Extrinsic/equipment & $\begin{array}{l}\text { Center } \\
\text { frequency }\end{array}$ & $f$ & $\mathbf{k H z}$ & {$[37,39]$} & {$[37.5,38.5]$} & \\
\hline Extrinsic/equipment & $\begin{array}{l}\text { Pulse } \\
\text { duration }\end{array}$ & $\boldsymbol{T}$ & ms & {$[0.5,1.5]$} & {$[0.5,1.5]$} & \\
\hline
\end{tabular}

solving an optimization problem, as elaborated in Ref. 5. According to this, the target strength of copper sphere with nominal target strength in the vicinity of $-35 \mathrm{~dB}$ is to remain as constant as possible with respect to variations in medium temperature. It is in this sense that the specified sphere is optimal.

Variations in the parameters are performed over ranges encompassing those of likely encounter. These are summarized in Table I. The computational ranges easily exceed those of likely encounter for each intrinsic parameter. This is evident for the two sound speeds, for instance, for their ordinary ranges are probably much narrower than the extreme limits prescribed by individual studies. According to the comprehensive collation of Ledbetter and Naimon for cop$\operatorname{per}^{16}$ and the usual elastic relations, ${ }^{17}$ the likely range of the longitudinal sound speed is $[4.66,4.80] \mathrm{km} / \mathrm{s}$ and for the transverse sound speed $[2.22,2.28] \mathrm{km} / \mathrm{s}$, assuming a density of $8.95 \mathrm{~g} / \mathrm{cm}^{3}$.

The ranges in medium parameters are determined from the usual hydrographic relations ${ }^{9,10}$ over the temperature range from 0 to $30^{\circ} \mathrm{C}$ and salinity range from 0 to $35 \mathrm{ppt}$, as applied at the surface, precluding pressure effects. The temperature range is identical with that used in determining the diameter of the sphere, which can thus be used in global marine applications.

Ranges of variation in the equipment parameters are defined quite liberally. These simulate both untuned conditions of operation and, in the case of the pulse duration, perhaps alternatives to the reference duration of $1 \mathrm{~ms}$.

Results are expressed through the absolute area units of backscattering cross section and through the logarithmic units of target strength, ${ }^{18}$ where reference is made to the
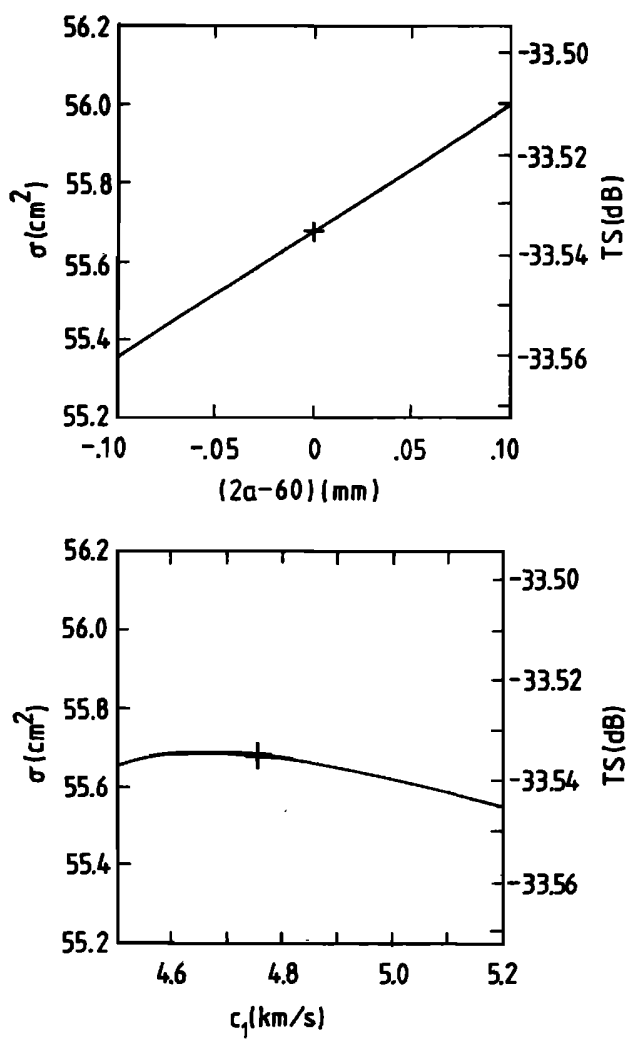
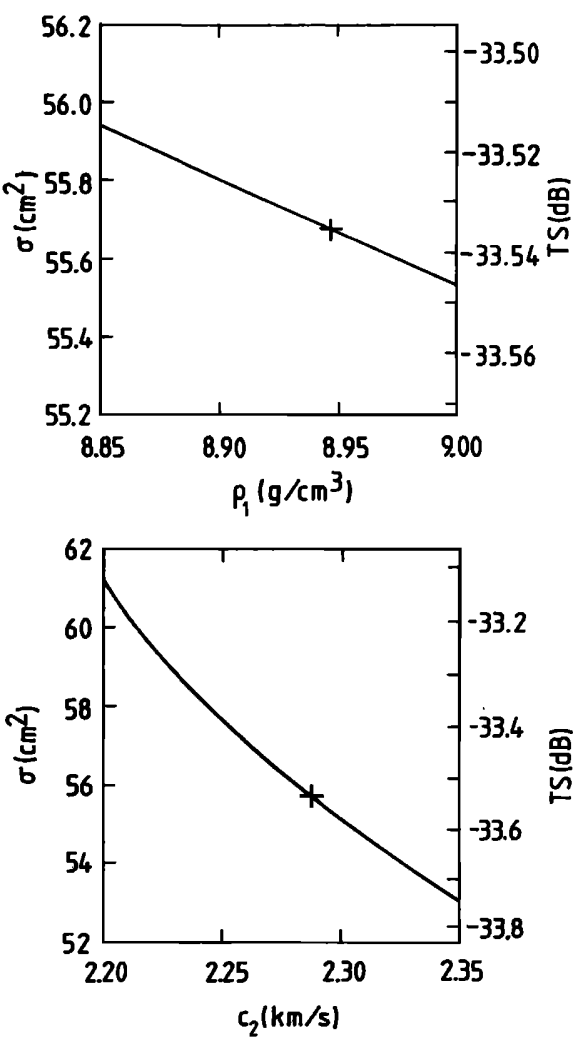

FIG. 1. Effects of variations in four intrinsic parameters on the backscattering cross section $\sigma$ and target strength TS of the optimal 60-mm-diam copper sphere used to calibrate $38-\mathrm{kHz}$ echo sounders. The sphere diameter is denoted by $2 \mathrm{a}$, density $\rho_{1}$, longitudinal sound speed $c_{1}$, and transverse sound speed $c_{2}$. The plus indicates the numerical baseline or constant reference common to all computations of the paper. 

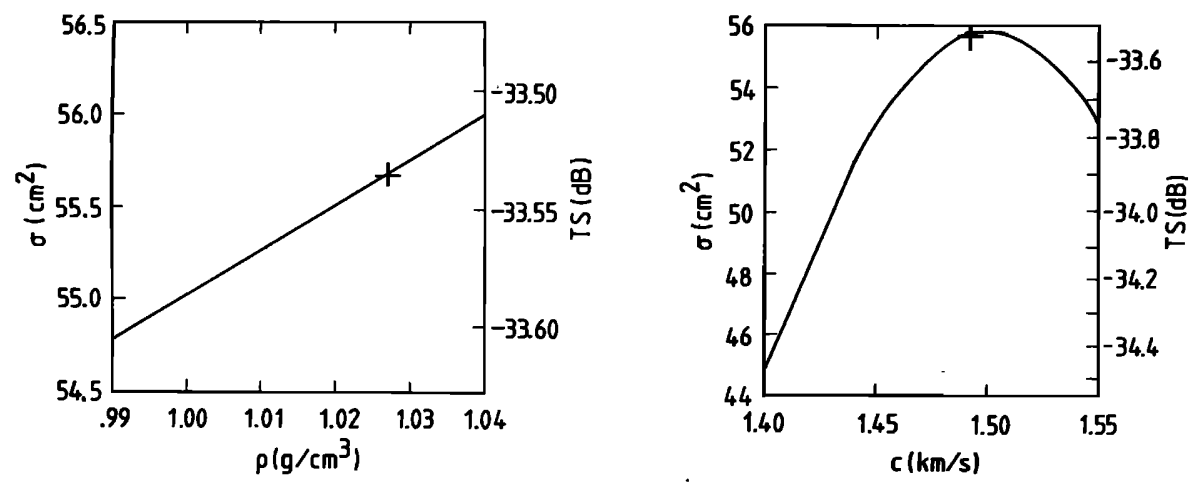

FIG. 2. Effects of variations in the medium density $\rho$ and sound speed $c$ on $\sigma$ and TS of the examined calibration sphere. The plus indicates the numerical baseline.

idealized perfectly reflecting sphere of 2-m radius with target strength of $0 \mathrm{~dB}$. In showing the various dependences of these quantities, the varied parameters or dependent variables are displayed in absolute units, as being preferable to relative units for applications.

\section{RESULTS AND DISCUSSION}

The results of performing the variations of Table I are given in Figs. 1-4 and in Table II. During the variations of each parameter, the other parameters maintained their constant reference values except when the medium parameters were the subjects of the variation. These exceptional cases are explained below.

The basic accuracy of the numerical evaluations of Eq. (1) exceeds one part in five hundred or $0.01 \mathrm{~dB}$, although the exact limit has not been determined. This minimal accuracy is convenient for the example of the $60-\mathrm{mm}$ copper sphere used to calibrate $38-\mathrm{kHz}$ echo sounders: since its nominal backscattering cross section is $55.7 \mathrm{~cm}^{2}$, the accuracy of the computations is at least of the order of $0.1 \mathrm{~cm}^{2}$. This is an appropriate truncation level for use in computing variational effects through the equations of Table II.

When variations in the intrinsic parameters are considered, as in Fig. 1, the total variations in backscattering cross section $\sigma$ are seen to be rather small except in the case of the transverse sound speed. In fact, in the case of electrical-grade copper, which is proposed for precision calibrations, ${ }^{4,5}$ the variations in $\sigma$ due to deviations in diameter, density, or longitudinal sound speed from the nominal state are expected to be less than $0.1 \mathrm{~cm}^{2}$. This assumes a machining tolerance of $0.02 \mathrm{~mm}$, density variation of $0.02 \mathrm{~g} / \mathrm{cm}^{3}$, or essentially arbi-

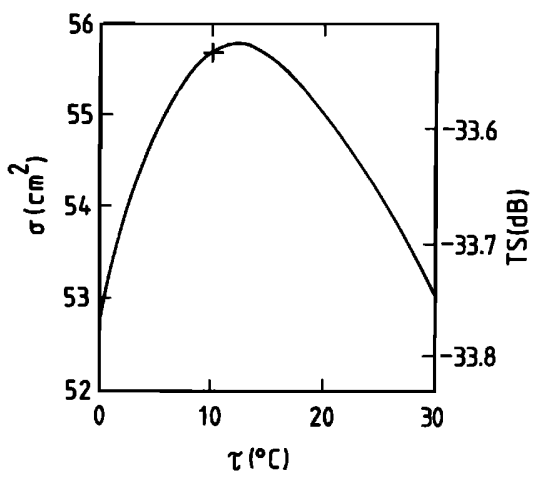

FIG. 3. Effects of variations in the medium temperature $\tau$ on $\sigma$ and TS of the examined calibration sphere. A constant medium salinity of $35 \mathrm{ppt}$ is assumed. The plus indicates the numerical baseline. trary variation in longitudinal sound speed. The author's experience with electrical-grade coppers confirms the practicability of these specifications.

The sensitivity of $\sigma$ to the transverse sound speed is well appreciated, especially in comparison with the influence of the longitudinal sound speed on $\sigma{ }^{1,19}$ In the present case, an unknown variation in $c_{2}$ of $0.03 \mathrm{~km} / \mathrm{s}$, that given in Ref. 16 for all copper types, would introduce an uncertainty of about $1.5 \mathrm{~cm}^{2}$ or $0.1 \mathrm{~dB}$. This could jeopardize a precision calibration, although, of course, $c_{2}$ is knowable with high accura$c y,{ }^{19,20}$ so that an uncertainty need not be tolerated in any practical application.

Treatment of the medium density and sound speed as independent parameters in Fig. 2 is somewhat contrived, since they are linked through the basic hydrographic parameters of temperature and salinity., ${ }^{9,10}$ The displayed variations are useful, nonetheless, for they illustrate the importance of accounting for the precise hydrographic conditions in performing precision calibrations.

This conclusion is reinforced by Fig. 3. In computing the temperature dependence of $\sigma$, the temperature has been incorporated in Eq. (1) through the medium density and sound speed in the monochromatic backscattering amplitude $F$. Each of the intrinsic parameters also depends on the temperature, but the coefficient of expansion ${ }^{7}$ and elasticity constants ${ }^{8}$ vary only slightly over the examined $30^{\circ} \mathrm{C}$ range in temperature, hence are neglected. For the same temperature range and the assumed constant salinity of $35 \mathrm{ppt}$, the temperature-induced variation in $\sigma$ is roughly quadratic, with peaking about $12^{\circ} \mathrm{C}$. This behavior reflects the aforementioned manner of determining the sphere diameter by optimization. The total variation in $\sigma$ is seen to approach 3 $\mathrm{cm}^{2}$ or $0.3 \mathrm{~dB}$, which is unimportant since the hydrography can always be measured and the appropriate corrections made.

It is to be noted in the present context that the $60-\mathrm{mm}$ copper sphere was designed for a standard marine echo sounder, normally used and calibrated in sea water of nominal salinity of $35 \mathrm{ppt}$. Extreme variations in the sound speed would not be expected to lie outside of the range $[1.45,1.55]$ $\mathrm{km} / \mathrm{s}$, for which the total variation in $\sigma$, as noted, does not exceed $3 \mathrm{~cm}^{2}$. Were the same or similar equipment to be calibrated in fresh water over the same $30^{\circ} \mathrm{C}$ temperature range, then the sound speed would lie in the range [1.40, $1.50] \mathrm{km} / \mathrm{s}$. The total range of $\sigma$ could then exceed $10 \mathrm{~cm}^{2}$ or nearly $1 \mathrm{~dB}$ according to Fig. 2 . This is dangerously large for 

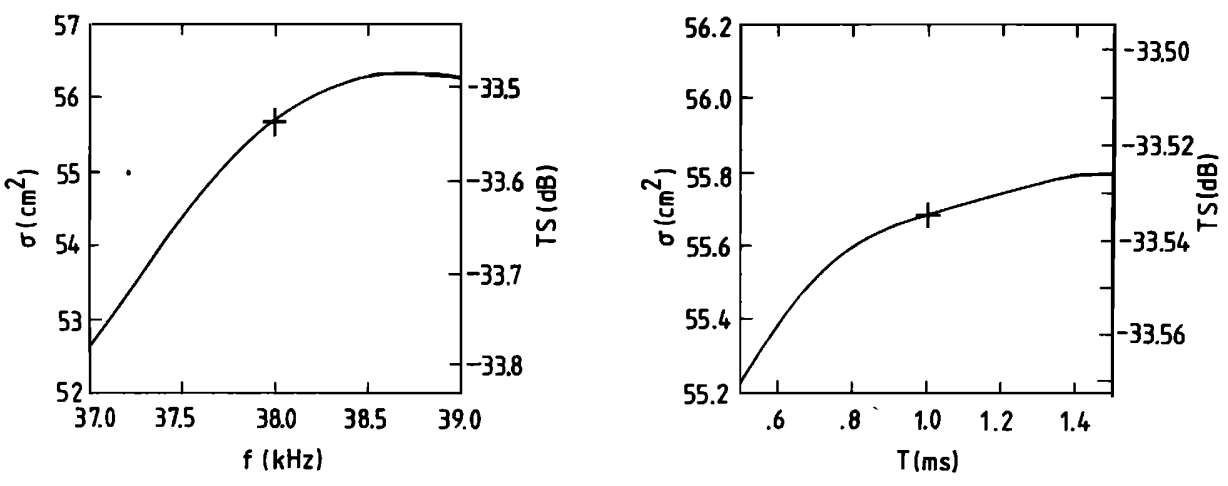

FIG. 4. Effects of variations in the center frequency $f$ and duration $T$ of the transmit pulse on $\sigma$ and TS of the examined calibration sphere. The plus indicates the numerical baseline.

precision calibrations, whether or not the exact hydrographic state is determined. Thus were the $38-\mathrm{kHz}$ echo sounder of the example to be used and calibrated in fresh water, then the optimal sphere diameter should be determined anew.

Equipment parameters can generally be controlled with very high precision, hence the variations shown in Fig. 4 pose no threat at all to precision calibrations. They indicate, however, how the backscattering cross section may be adjusted when the equipment is untuned, or perhaps used at an off-frequency or with different pulse duration than that chosen for the baseline.

The frequency response function of the receiver also influences the backscattering cross section as an observable. ${ }^{5}$ For the present study, the receiver characteristic of the Simrad EK-400/38 echo sounder has been used. Computations performed using the characteristics of other $38-\mathrm{kHz}$ echo sounders, for example, the EK-38 system, show nearly identical results, with possible differences lying under $0.1 \mathrm{~cm}^{2}$ or $0.01 \mathrm{~dB}$.

Although only the effects of single-parameter variations have been studied quantitatively here, the effects of multiple-parameter variations may be deduced from these in a number of cases. The backscattering cross section $\sigma$, as defined in Eq. (1), is analytic for the conditions of the numerical example. It may thus be expanded in a generalized Tay- lor's series in the varied parameters. Whenever the effects of these on $\sigma$ are linear, the influence of simultaneous variations of several parameters may be determined by simple compounding or addition of the effects. Thus, for example, the ratio of backscattering cross sections of two copper spheres differing only by their diameters, which, however, lie in the range $[59.9,60.1] \mathrm{mm}$, will remain constant for arbitrary variations in the sphere density, longitudinal sound speed, medium density, and pulse duration, over the broad ranges specified in Table I. The same ratio will remain constant for variations of the other parameters when linear in the vicinity of the baseline, for example, for the transverse sound speed in the range $[2.23,2.30] \mathrm{km} / \mathrm{s}$ or for the center frequency in the range $[37.8,38.1] \mathrm{kHz}$, where computations have shown constancy to within $10 \%$. The effects of temperature, hence medium sound speed too, are intrinsically nonlinear, and linear compounding with the effect of variation of a second parameter can succeed only over minute ranges of the nonlinear parameter.

\section{CONCLUSIONS}

The effects of parameter variations on the backscattering cross section of an optimal copper calibration sphere have been determined quantitatively for nine different variables representing properties or characteristics of the sphere,

TABLE II. Algebraic representations of the effects of single-parameter variations on the backscattering cross section, given the numerical baseline defined in the text. Computational results should be rounded to the nearest $0.1 \mathrm{~cm}^{2}$.

\begin{tabular}{|c|c|c|c|c|}
\hline Parameter & Units & Backscattering cross section $\sigma\left(\mathrm{cm}^{2}\right)$ & $\begin{array}{l}\text { Range of } \\
\text { applicability }\end{array}$ & $\begin{array}{l}\text { Precision } \\
\left(\mathrm{cm}^{2}\right)\end{array}$ \\
\hline $2 a$ & $\mathrm{~mm}$ & $55.68+3.2(2 a-60)$ & {$[59.9,60.1]$} & 0.01 \\
\hline$\rho_{1}$ & $\mathrm{~g} / \mathrm{cm}^{3}$ & $55.95-2.7\left(\rho_{1}-8.85\right)$ & {$[8.85,9.00]$} & 0.01 \\
\hline \multirow[t]{3}{*}{$c_{1}$} & $\mathbf{k m} / \mathbf{s}$ & $55.69-0.16\left(c_{1}-4.5\right)$ & {$[4.5,5.2]$} & 0.04 \\
\hline & & $\stackrel{\text { or }}{55.66}+0.3\left(c_{1}-4.5\right)$ & {$[4.5,4.6]$} & \\
\hline & & $\left\{\begin{array}{l}55.69 \\
55.69-0.3\left(c_{1}-4.75\right)\end{array}\right.$ & $\begin{array}{l}{[4.6,4.75]} \\
{[4.75,5.2]}\end{array}$ & 0.01 \\
\hline \multirow[t]{2}{*}{$c_{2}$} & $\mathbf{k m} / \mathbf{s}$ & $\begin{array}{l}60.8-54\left(c_{2}-2.2\right) \\
\text { or }\end{array}$ & {$[2.2,2.35]$} & 0.4 \\
\hline & $\mathrm{g} / \mathrm{cm}^{3}$ & $\begin{array}{l}60.5-55\left(c_{2}-2.2\right)+140\left(c_{2}-2.27\right)^{2} \\
54.79+24(\rho-0.99)\end{array}$ & $\begin{array}{l}{[2.2,2.35]} \\
{[0.99,1.04]}\end{array}$ & $\begin{array}{l}0.1 \\
0.01\end{array}$ \\
\hline \multirow[t]{2}{*}{$c$} & $\mathbf{k m} / \mathbf{s}$ & $55.77-1200(c-1.5)^{2}$ & {$[1.40,1.55]$} & 1.1 \\
\hline & & {$\left[0.021(\tau-12)^{2}\right.$} & $l_{[0,12}[1.45,1.55]$ & 0.2 \\
\hline$\tau$ & ${ }^{\circ} \mathbf{C}$ & $55.77-\left[\begin{array}{l}0.021(\tau-12) \\
0.0086(\tau-12)^{2}\end{array}\right.$ & $\begin{array}{l}{[0,12]} \\
{[12,30]}\end{array}$ & 0.2 \\
\hline$f$ & $\mathbf{k H z}$ & $54.55+1.9(f-37.5)$ & {$[37.5,38.5]$} & 0.2 \\
\hline$T$ & ms & $55.62+0.35(T-0.8)$ & {$[0.8,1.2]$} & 0.02 \\
\hline
\end{tabular}


immersion medium, and equipment. In many cases the effects are seen to be quite small. That is, the dependences of the backscattering cross section on the parameters are weak. Unknown or uncompensated variations in such parameters cannot upset a precision calibration, or one whose accuracy is $0.1 \mathrm{~dB}^{5}$

In all conceivable cases where optimal copper spheres are used, the effects of parameter variations are calculable. Measurement of the exact conditions of performance of any particular calibration will thus enable the appropriate value of backscattering cross section to be determined. Since the magnitudes of the effects are known to one part in five hundred or $0.01 \mathrm{~dB}$, the possibility of maintaining precision calibrations under quite variable conditions is evident.

The possibility of using copper spheres for the measurement of high-intensity fields by the radiation force method $^{21,22}$ is also apparent. As noted in Refs. 23 and 24, there are many other candidates for the particular application, but as noted in Ref. 5, copper is an especially favorable material for many reasons. Its full potential in the precision calibration of acoustic equipment is yet to be realized.

\section{ACKNOWLEDGMENTS}

The author acknowledges particular indebtedness to his colleagues G. Vestnes and H. P. Knudsen, both of the Institute of Marine Research, for their emphasis of the very practical, and very crucial, turning points of calibration procedures. The same colleagues' reviews of earlier manuscripts by the author have been so thorough as to render the appendix a joint effort. This was recognized through the coauthorship of Ref. 29, the source of the appendix.

\section{APPENDIX: PASSIVE TARGET CALIBRATION}

This section is offered to explicate the method of passive target calibration by a very practical example: that of calibration of echo sounders and integrators on board oceangoing research vessels. A standard procedure is outlined. Maintenance of 0.1-dB precision is described, as earlier, for a wide range of temperatures and for untuned equipment. Specific reference is made to a calibration exercise of the Norwegian research vessel MichaEL SARS. The long-term stability of quoted, cumulative calibration results for this ship witnesses to the reliability and precision of the method.

\section{A. Introduction}

In essence, calibration of hydroacoustic equipment by spherical targets is simple. The target is placed at a known position in the transducer beam, ensonified, and the properties of the echo related to the known scattering characteristics of the target. Specification of the relationship between the target and response of the acoustic equipment, including signal processing hardware and software, constitutes the calibration.

In practice, however, calibration is seldom a simple matter. Getting a 1-kg, 60-mm-diam sphere to hang virtually motionless in the center of an $8 \mathrm{deg}$ beam, $20 \mathrm{~m}$ beneath the hull of a 1500-ton research vessel is not undertaken light- ly. Yet the success of this operation is absolutely essential to even an ordinary calibration, not to mention precision calibration with expected 0.1-dB accuracy.

Appreciation of this fact has prompted the present contribution, a sequel to the rather obscure Ref. 4. To remedy the apparent lack of detailed accounts of calibration procedures in the general or easily accessible literature, the method of calibration with spherical targets is described in detail. To encourage performance of precision calibration whenever opportunity permits, the particular application of optimal copper spheres to the routine is elaborated. The abstractions of the method and theory are offset by a practical example: calibration of $R / V$ MICHAEL SARS. Data from both an individual calibration exercise and from the semiannual series of calibration exercises are presented. In addition commenting on these, the problem of intercalibration and the improvement of present procedures are discussed.

\section{B. The method}

The general process of large-system calibration is now described. This is oriented towards the ocean-going research vessel, but may be applied whenever and wherever echo sounders or echo integrators must be calibrated.

\section{Preliminary performance measurement and adjustment of equipment}

Six basic tasks are enumerated here. These should precede the physical part of the calibration. They may be conducted expeditiously while the vessel is underway, for example, while sailing to its calibration anchorage.

(i) Measure and record transducer insulation and impedance.

(ii) Check and adjust, as necessary, the mains voltage and all critical voltages in the transmitter and receiver.

(iii) Check and record the two time-varied-gain functions; namely, the $20 \log r+2 \alpha r$ and $40 \log r+2 a r$ functions.

(iv) Check and record total gains, bandwidths, and accuracy of the -10 - and $-20-\mathrm{dB}$ attenuators.

(v) Measure and record the various effects for all combinations of transmitters and pulse durations of common or possible use during the preceding or following cruise program for which the calibration is being undertaken.

(vi) Confirm the echo integrator performance by playing in a known signal and observing, hence recording, the output. Adjust the electronics as necessary to meet specifications. Confirm the vessel log compensation.

\section{Rigging for sphere measurements}

The vessel is anchored in calm and sheltered water. The depth must be sufficient for separation of sphere and bottom echoes. It is desirable, moreover, to work in water as deep as possible, consistent with maintaining a stable platform. Both bow and stern anchoring or tying are recommended. This is illustrated in Fig. A1.

Winches to guide and steer lines to the sphere for its centering in the echo sounder beam are affixed to the deck railing. This is done in accordance with detailed ship draw- 


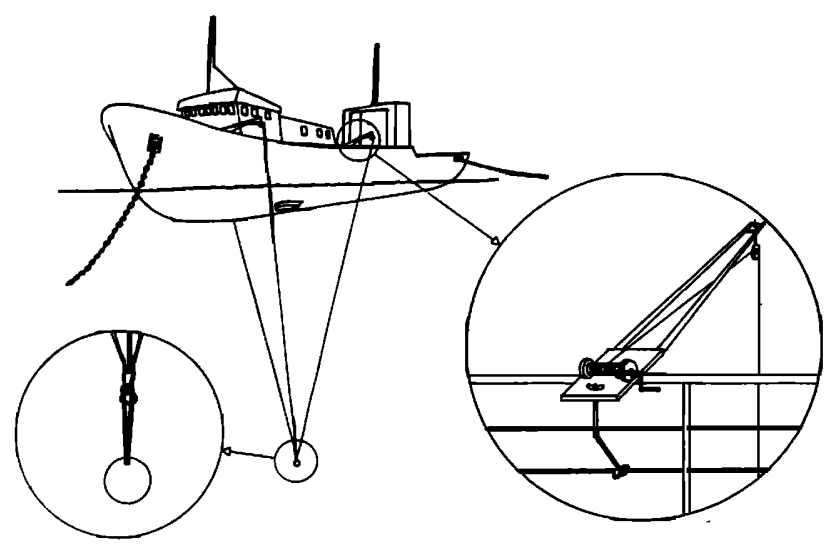

FIG. A1. Rigging of a research vessel for calibration.

ings. The first winch is placed in the transverse plane of the ship running through the transducer. The second and third winches are placed on the opposite boat side and at equal distances from the transverse section containing the transducer and first winch. Each winch is provided with a long spool of $0.60-\mathrm{mm}$-diam monofilament nylon, which is marked with small lead weights at 5-m intervals, beginning $10 \mathrm{~m}$ from the loose end.

Prior to commencing the sphere measurements, the lines from the two winches on the same side of the boat are drawn beneath the hull to the other winch by means of a line passed under the keel before anchoring. The appropriate sphere, with affixed loop, is attached to the three suspension lines, cf. Fig. A1. It is then immersed in a solution of soap and fresh water and lifted overboard by the fastened lines without being touched. The sphere is lowered beneath the vessel to the desired depth, for example, $25 \mathrm{~m}$, which is determined roughly by counting the lead marker-weights on each line.

The sphere depth or range from the transducer is determined by several considerations. The minimal allowable range to the sphere is the greater of the Rayleigh distance, or square of the largest transducer dimension divided by the acoustic wavelength, which defines the nearfield/farfield transition, and the least range for which the sphere echo does not saturate the electronics, e.g., the preamplifier. Two further practical considerations in choosing the range are the transducer beamwidth and vessel geometry. The physical width of the beam, which increases linearly with range, should be sufficiently great so that the sphere echo is unaffected by the small, perhaps pendular movements to which it is inevitably subjected. The minimal range must also be convenient with respect to the vessel geometry. In particular, if the suspension lines do not hang freely, then control of the sphere may be hindered by friction or possible obstructions on the hull. Despite the number and variety of these considerations, it is seldom difficult in practice to find a suitable range which satisfies all of the above criteria.

\section{Hydrography}

During the anchoring and rigging operations, the temperature and salinity profiles should be taken. These will allow computation of the sound speed both at discrete depths and cumulatively to the depths of possible sphere suspension. The second computation will allow determination of the exact depth of eventual sphere suspension from the echo time delay. When this depth is applied in the first computation, the temperature correction to the target strength of the calibration sphere may be obtained from a reference graph or table.

\section{Centering}

The purpose of this crucial operation is to move the immersed, suspended sphere onto the acoustic axis of the transducer. Movement of the sphere occurs by turning of the various hand-winches, always singly and upon specific command by the director of this procedure, who is guided by constant observation of the echo waveform on the oscilloscope. The two principles guiding the search for the beam center are (i) preliminary exploration of the beam to ensure location of the sphere in the mainlobe, and (ii) further probing to find the position of strongest echo. In the case of highly directional transducers, determination of the ultimate axial location is made when any movement of any winch, in or out, cannot increase the echo amplitude.

\section{Computations for echo sounder calibration}

The sphere range is measured in units of milliseconds from the echo on the oscilloscope. The range $r$ in meters is then given by $r=c t / 2$, where $t$ is the measured echo time delay and $c$ is the average speed of sound from transducer to sphere depth. The approximate root-mean-square $(\mathrm{rms})$ echo amplitude $u_{\text {rms }}$ is determined from the peak-to-peak amplitude $u_{\mathrm{pp}}$ by assumption of a rectangular echo pulse, hence $u_{\mathrm{rms}}=u_{\mathrm{pp}} /\left(2 \cdot 2^{1 / 2}\right)$. This is converted to logarithmic units by the definition: $U=20 \log u_{\mathrm{rms}}$, where $u_{\mathrm{rms}}$ is expressed in volts. The sum of source level SL and voltage response VR is now determined from the target strength TS of the calibration sphere, after appropriate fine adjustments. The specifying equations are

$\mathrm{SL}+\mathrm{VR}=U_{1}-\mathrm{TS}+20 \log R_{1}+2 \alpha R_{1}-G+20 \log r$

for "20 $\log r$ " TVG, and

$$
\mathrm{SL}+\mathrm{VR}=U_{2}-\mathrm{TS}+40 \log R_{2}+2 \alpha R_{2}-G
$$

for " $40 \log r$ " TVG, where $U_{1}$ and $U_{2}$ are the respective echo levels with the two TVG functions, $r$ is the sphere range, $R_{1}$ and $R_{2}$ are the respective cutoff ranges of the TVG functions, $\alpha$ is the absorption coefficient used in the functions, and $G$ is the attenuator setting. The units of the various quantities are stated in Table AI.

\section{Readiness of echo integrator}

The echo sounder is adjusted to its normal cruise settings. For the Simrad EK-38 echo sounder, for example, these might be the following: selected transducer: $30 \times 30$, transmitter: external, pulse duration: $0.6 \mathrm{~ms}$, TVG: "20 $\log r$ ", attenuator setting: $-20 \mathrm{~dB}$, bandwidth: $3 \mathrm{kHz}$, range scale: $0-250 \mathrm{~m}$. The vessel speed is simulated as 10 knots and the observation time chosen to be six minutes, 
TABLE AI. Units of quantities in calibration equations.

\begin{tabular}{lll}
\hline \hline Quantity & Symbol & Units \\
\hline Source level & $\mathrm{SL}$ & $\mathrm{dB} r e 1 \mu \mathrm{Pa}$ \\
Voltage response & $\mathrm{VR}$ & $\mathrm{dB} r e 1 \mathrm{~V} / \mu \mathrm{Pa}$ \\
Echo level & $U_{1}, U_{2}$ & $\mathrm{~dB} r e 1 \mathrm{~V}$ \\
Target strength & $\mathrm{TS}$ & $\mathrm{dB}$ \\
Ranges & $r, R_{1}, R_{2}$ & $\mathrm{~m}$ \\
Absorption coefficient & $\alpha$ & $\mathrm{dB} / \mathrm{m}$ \\
Gain or attenuator setting & $G$ & $\mathrm{~dB} r e 1 \mathrm{~V}$ \\
& & \\
\hline \hline
\end{tabular}

corresponding to a sailed distance of $1 \mathrm{nmi}$. Three 5-m-thick echo integration channels or layers are defined: the central channel is centered at the sphere depth and the others are placed immediately above and below, thus sharing common limits with the central channel. No threshold is used. The middle channel will thus contain the contribution from the sphere echo; the others will measure noise including volume reverberation.

\section{Computations for echo integrator calibration}

The echo energy from each of a large number of pings is computed. The largest of these, if within about $10 \%$ of the average, is extracted. If the deviation is larger, then the centering operation should be repeated and the acoustic measurements performed anew. The product of the largest echo energy finally selected and the total number of pings in its sequence is expressed in terms of the arbitrary, historical units of millimeters of pen deflection on a strip-chart recorder per meter of sailed distance at 10-knots speed and the ping rate of most common use, and for the given target referred to 1-m range. Despite the a priori oddity of this conversion, the fact of all pertinent calibration data being stored guarantees the possibility of intercalibration with other echo integrators, on other research vessels, by citation of the peak echo energy, for example.

\section{Documentation}

Special preprinted forms are filled in during the entire course of measurements. Collateral documentation in the form of oscilloscope photographs and hydrographic measurements are attached to these forms, all of which are identified for future reference. Copies are left on board, distributed otherwise as necessary, and the originals are filed in the archives of the institute.

\section{Preserving precision}

As mentioned above, the calibration value of target strength is adjusted for the temperature. In more general terms, the target strength depends on the hydrography, or temperature and salinity of the immersion medium at the depth of sphere suspension. This is clearly seen in Faran's paper, ${ }^{6}$ as well as in many later works, for example, Refs. 1 and 25-27. In each of these, the hydrographic dependence appears implicitly through the density and sound speed of the medium. The connection between these parameters and the hydrography are well known., ${ }^{9,10}$

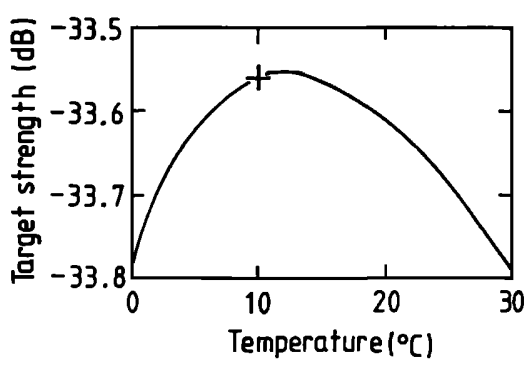

FIG. A2. Effects of temperature variations on the target strength of the 60mm copper sphere used for calibration of the Simrad EK-38 echo sounder. The plus sign indicates a common datum with the other computations, which are represented here by the constant center frequency of $38 \mathrm{kHz}$, pulse duration of $0.6 \mathrm{~ms}$, and temperature of $10^{\circ} \mathrm{C}$.

The influence of temperature on the target strength of the $60-\mathrm{mm}$ copper sphere has already been investigated, both above and in Ref. 28. The result of this is presented in Fig. A2 for marine calibrations of the Simrad EK-38 echo sounder when operated under the following nominal conditions: $38-\mathrm{kHz}$ center frequency, $0.6-\mathrm{ms}$ pulse duration, and $3-\mathrm{kHz}$ receiver bandwidth. The temperature is varied from $0^{\circ}$ to $30^{\circ} \mathrm{C}$, assuming a constant salinity of $35 \mathrm{ppt}$.

Evidently, use of a target strength of $-33.7 \mathrm{~dB}$ will ensure a precision calibration over the entire $30^{\circ} \mathrm{C}$ range, without requiring temperature compensation. This is a direct consequence of the method of determining the sphere diameter, given its composition of electrical-grade copper. ${ }^{4,5}$ Application of the temperature-corrected target strength will, however, contribute to the overall control of potential calibration errors, hence is recommended in the general case. In the present case, use of the nominal target strength of $-33.6 \mathrm{~dB}$ for temperatures from $4^{\circ}$ to $22^{\circ} \mathrm{C}$ will assist this control, as the single value of $-33.7 \mathrm{~dB}$ does not make allowance for rounding errors.

Both the center frequency and duration of the transmit pulse can also influence the calibration value of target strength. ${ }^{5}$ These dependences are shown in Figs. A3 and A4, respectively, for the following nominal hydrographic state: temperature of $10{ }^{\circ} \mathrm{C}$ and salinity of $35 \mathrm{ppt}$, hence medium density of $1027 \mathrm{~kg} / \mathrm{m}^{3}$ and sound speed of $1490 \mathrm{~m} / \mathrm{s}$.

Again, as with the temperature, the dependences are weak, and use of the nominal value, $-33.6 \mathrm{~dB}$ after rounding, would permit neglect of variations in the two parameters over the considered ranges. However, since both equipment

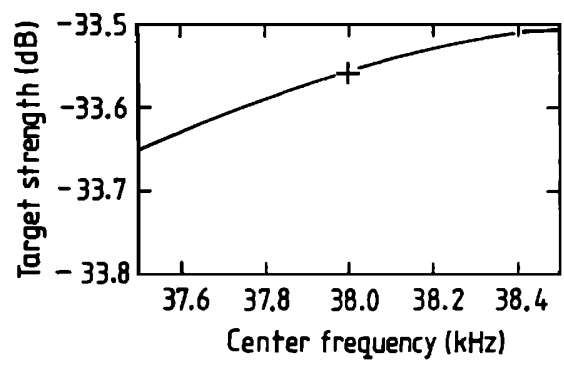

FIG. A3. Dependence of the target strength of the $60-\mathrm{mm}$ copper sphere on the transmitter center frequency of the EK-38 echo sounder. The plus sign indicates a common datum with Figs. A2 and A4. 


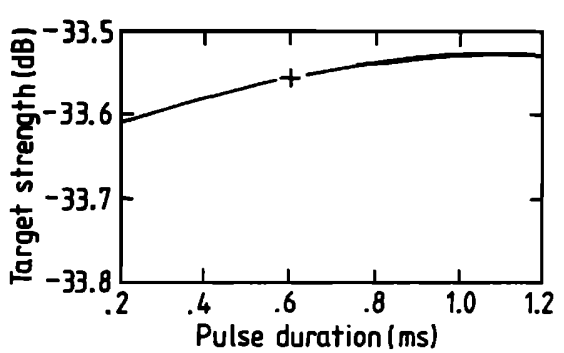

FIG. A4. Dependence of the target strength of the 60-mm copper sphere on the duration of the transmit pulse of the EK-38 echo sounder. The plus sign indicates a common datum with Figs. A2 and A3.

parameters can be measured with high accuracy, control of the calibration process would dictate adjustment of the target strength.

It might be thought that it is best to maintain a tuned condition for the sake of calibration. In fact, this is unnecessary, for the acoustic robustness of optimal copper spheres, a consequence of their design by optimization, allows their use over a wide range of conditions departing from nominality. Thus equipment that is discovered to be out of tune or that cannot be tuned easily, because of age, for instance, can be calibrated by the same sphere by a slight modification in the target strength.

\section{Example}

The method of calibration is illustrated by an example derived from the exercise with R/V MichaEL SARS on 5 January 1982. This concerns the Simrad EK-38R echo sounder and attached echo integrator, standard equipment videly used in the acoustic estimation of fish abundance.

The exercise began at 0800 hours with the departure of the boat for Skogsvaagen, an inlet of the island of Sotra, about a one-hour sail from Bergen, where the sphere measurements were to be conducted. While underway, the performance of the equipment was measured. This followed the task list of item (1) of the method. No serious deviations from the specifications were discovered, precluding special adjustments. In addition to these preparatory measurements, the three outriggers with hand-winches were attached to the deck railing in their usual positions and the several copper target spheres were immersed in a bucket of fresh water and detergent.

At Skogsvaagen the boat was anchored near the northern shoreline of the inlet, hugging a rock wall affording shelter from a light north breeze. Both bow and stern were anchored in water of $100-\mathrm{m}$ depth. Owing to local geography, tidal flow and other submarine currents are completely negligible in the inlet, hence were not a consideration in choosing the anchoring location.

The hydrography was performed immediately upon completion of the anchoring. The temperature and salinity profiles measured by a standard CTD-sonde were logged automatically by the central computer and computations of sound speed and density performed. At the anticipated calibration depth of $24 \mathrm{~m}$, the temperature and salinity were found to be about $6^{\circ} \mathrm{C}$ and $33 \mathrm{ppt}$, implying a local sound speed of $1472 \mathrm{~m} / \mathrm{s}$ and density of $1026 \mathrm{~kg} / \mathrm{m}^{3}$. The average sound speed from the transducer to this depth was found by computation to be $1466 \mathrm{~m} / \mathrm{s}$.

Calibration of the 38-kHz equipment generally has the highest priority in this kind of exercise, hence the $60-\mathrm{mm}$ copper sphere was immersed first. Its echo was observed on the oscilloscope immediately upon lowering to approximate 24-m depth, suggesting its location in the mainlobe. This was confirmed by routine exploration of the beam.

After fine adjustment, the sphere was assumed to be on the acoustic axis of the transducer and the measurements were begun. These are now described for the "20 $\log r$ " TVG function and external transmitter, the standard combination for many acoustic surveys. The sequences of items (5) and (7) of the method are followed below.

The echo time delay $t$ was measured as $25.2 \mathrm{~ms}$ on the oscilloscope. Use of the average sound speed $c=1466 \mathrm{~m} / \mathrm{s}$ determined the sphere range $r=c t / 2=18.5 \mathrm{~m}$. The peakto-peak sphere echo $u_{\mathrm{pp}}$ was measured with the attenuator setting or gain $G=-20 \mathrm{~dB}$ with the result $u_{\mathrm{pp}}=3.35 \mathrm{~V}$. This was converted to the echo level $U_{1}=20 \log u_{\mathrm{pp}} /$ $\left(2 \cdot 2^{1 / 2}\right)=1.5 \mathrm{~dB}$. The cutoff range $R_{1}$ of the " $20 \log r$ " TVG function is $502 \mathrm{~m}$. The absorption coefficient $\alpha$ for the particular echo sounder is $0.0105 \mathrm{~dB} / \mathrm{m}$. For the exact sphere depth, the hydrography dictated a target strength TS $=-33.6 \mathrm{~dB}$, cf. Fig. A2. The sum of the source level SL and voltage response VR can now be determined:

$$
\begin{aligned}
\mathrm{SL}+\mathrm{VR} & =U_{1}-\mathrm{TS}+20 \log R_{1}+2 \alpha R_{1}-G+20 \log r \\
& =1.5+33.6+54.0+10.5+20.0+25.3 \\
& =144.9 \mathrm{~dB} .
\end{aligned}
$$

In practice, reference is generally made to the actual attenuator setting of the measurement, which is also that of greatest use in survey work; namely, $G=-20 \mathrm{~dB}$. According to this reference, $S L+V R=124.9 \mathrm{~dB}$.

Following calibration of the echo sounder, the echo integrator was prepared for its calibration. This was performed in the manner described in item (6) of the method. Because of the sphere range of $18.5 \mathrm{~m}$, the central 5-m-thick integration volume was defined as $[17,22] \mathrm{m}$. The adjacent channels were defined as $[12,17]$ and $[22,27] \mathrm{m}$.

The results of integrating the sphere echo for six minutes at the standard ping rate of $\mathbf{4 8}$ pings per minute are the following: average echo energy of $6934 \mathrm{~mm}$ and maximum echo energy of $7356 \mathrm{~mm}$. The observed excursion of $6 \%$ was considered acceptable. Further evidence for the acceptability of the measurement was provided by the measurements of echo energy, viz. reverberation, in each of the adjacent channels. The peak echoes lay between 10 and $20 \mathrm{~mm}$, i.e., about 25 to $30 \mathrm{~dB}$ below the sphere echo, which is typical. It was concluded from these measurements that there were no extraneous scatterers such as fish in the integration volume. This was also confirmed by observation of the oscilloscope during the integration: the sphere echo appeared entirely stable.

Calibration of the echo integrator can now be completed by reference of the peak echo energy to a 1-m-sailed distance and 1-m depth. The calibration value is expressed thus: 
TABLE AII. Summary of calibrations of the Simrad EK-38 echo sounder and attached echo integrator on board R/V MichAEL SARS.

\begin{tabular}{lll}
\hline \hline Date of exércise & $\begin{array}{l}\text { SL + VR } \\
\text { (dB) }\end{array}$ & $\begin{array}{l}\text { Integral } \\
\text { (mm) }\end{array}$ \\
\hline \hline January 1980 & 125.5 & 1387 \\
July 1980 & 124.9 & 1264 \\
January 1981 & 124.6 & 1291 \\
June 1981 & 124.9 & 1330 \\
January 1982 & 124.9 & 1359 \\
\hline \hline
\end{tabular}

Integral (mm re 1-m-sailed distance-1-m-depth)

$$
=\frac{7356 \cdot 18.5^{2}}{1852 \mathrm{~m} / \mathrm{nmi}}=1359 \text {. }
$$

Measurements of the source level and voltage response of the echo sounder were also made for other equipment settings; namely, for other transmitters for both the " $20 \mathrm{log}$ $r$ " and " $40 \log r$ " TVG functions. Documentation was collected, copied, and the originals deposited at the Institute upon completion of the cruise on the same day.

The derived numbers are compared with previous calibration results for the same boat in Table AII, where the sum of source level and voltage response is referred to the usual $-20-\mathrm{dB}$ attenuator setting. The consistency of corresponding numbers witnesses both to the precision of the calibrations and to the long-term stability of the equipment.

\section{E. Discussion}

Calibration of echo sounders and integrators is a straightforward process, but one that requires vigilance at all stages for its success. This evidently has been the case with calibration of the 38- $\mathrm{kHz}$ echo sounder and echo integrator of R/V Michael SARS, as illustrated in Table AII. A detailed analysis of the errors in a routine exercise has suggested a precision well under $0.5 \mathrm{~dB}$, if not approaching $0.1 \mathrm{~dB}$. The consistency of the tabulated numbers for the five cruises supports this, for the greatest excursion from the average sum of source level and voltage response for the echo sounder is $0.5 \mathrm{~dB}$, while the corresponding deviation in echo integral from the average is $5 \%$.

The present procedures can be improved, of course, and the Institute of Marine Research is continuing its work on this. In particular, measurement of the time-varied-gain function has proved to be problematical. To facilitate its measurement, special circuitry for performing the determination automatically is being designed. Eventually, it is planned to incorporate a TVG correction in the software of the echo integrator, to reduce what hardware errors may exist or develop with time.

Another procedure which could be improved is that of centering of the sphere. At present, the angular position of largest echo is sought. This is rather time consuming because of the relative broadness of the mainlobe, hence insensitivity of the echo to fine adjustments in position. Were it possible to operate the same or similar transducer in a split-beam mode, in which four quadrant beams are separately formed, then generation of the difference responses in the fore-and- aft and athwartships planes would enable very sensitive minima-ideally sharply defined nulls-to be sought. Not only would this accelerate the alignment process, but it would also effect a demonstrably highly accurate positioning. Additionally, observation of the sphere echo on the oscilloscope during calibration of the echo sounder would enable the data goodness to be confirmed without having to study the statistics.

When and if both improvements are made, it should be easy to establish the accuracy of calibrations of echo sounders and integrators. This is expected to approach 0.1 dB.

Intercalibration is entirely feasible given the present calibration procedures based on the use of optimal copper spheres. To determine the relative performances of two systems, either the same or similar spheres can be used. Different spheres could also be employed, if their target strengths were known with sufficient accuracy.

The significance of the present calibration method to multiple-vessel acoustic surveys of fish stocks is that intercalibration of the several instruments can be effected without requiring the vessels to meet and perform a simultaneous survey of the same fish aggregation. Use of optimal copper spheres is especially advantageous here, for the properties of copper as a standards material are unalloyed, and offer an immediate, potential accuracy of $0.1 \mathrm{~dB}$, with the possibility of further improvements.

'R. Hickling, "Analysis of echoes from a solid elastic sphere in water," J. Acoust. Soc. Am. 34, 1582-1592 (1962).

${ }^{2}$ R. T. Beyer, "A new wave of acoustics," Phys. Today 34 (11), 145-157 (1981).

${ }^{3}$ L. R. Dragonette, S. K. Numrich, and L. J. Frank, "Calibration technique for acoustic scattering measurements," J. Acoust. Soc. Am. 69, 11861189 (1981).

${ }^{4}$ K. G. Foote, H. P. Knudsen, G. Vestnes, R. Brede, and R. L. Nielsen, "Improved calibration of hydroacoustic equipment with copper spheres," Coun. Meet. Int. Coun. Explor. Sea 1981/B:20, Copenhagen, Denmark, 18 pp.

${ }^{5}$ K. G. Foote, "Optimizing copper spheres for precision calibration of hydroacoustic equipment," J. Acoust. Soc. Am. 71, 742-747 (1982).

6. J. Faran, Jr., "Sound scattering by solid cylinders and spheres," J. Acoust. Soc. Am. 23, 405-418 (1951).

${ }^{7}$ R. K. Kirby, T. A. Hahn, and B. D. Rothrock, "Thermal expansion," in American Institute of Physics Handbook (McGraw-Hill, New York, 1972), 3rd ed., p. 4-123.

${ }^{8}$ F. R. S. Hearmon, "The elastic constants of crystals and other anisotropic materials," in Landolt-Börnstein, New Series (Springer-Verlag, Berlin, 1979), Group III, Vol. 11, pp. 1-244.

${ }^{9} \mathrm{G}$. Dietrich, "Ozeanographie. Physikalische Eigenschaften des Meerwassers. Allgemeiner Teil," in Landolt-Börnstein Zahlenwerte und Funktionen (Springer-Verlag, Berlin, 1952), 6th ed., Vol. 3, p. 428.

${ }^{10}$ V. A. Del Grosso, "New equation for the speed of sound in natural waters (with comparison to other equations)," J. Acoust. Soc. Am. 56, 1084-1091 (1974).

${ }^{11}$ H. M. Trent, D. E. Stone, and R. B. Lindsay, "Density of solids," in American Institute of Physics Handbook (McGraw-Hill, New York, 1972), 3rd ed., p. 2-28.

${ }^{12}$ W. C. Overton, Jr. and J. Gaffney, "Temperature variation of the elastic constants of cubic elements. I. Copper," Phys. Rev. 98, 969-977 (1955).

${ }^{13}$ R. Hill, "The elastic behaviour of a crystalline aggregate," Proc. Phys. Soc. Sect. A, 65, 349-354 (1952).

${ }^{14}$ W. P. Mason "Acoustic properties of solids," in American Institute of Physics Handbook, (McGraw-Hill, New York, 1972), 3rd ed., p. 3-104. 
15J. R. Frederick, Ultrasonic Engineering (Wiley, New York, 1965).

${ }^{16} \mathrm{H}$. M. Ledbetter and E. R. Naimon, "Elastic properties of metals and alloys. II. Copper," J. Phys. Chem. Ref. Data 3, 897-935 (1974).

${ }^{17} \mathrm{H}$. Kolsky, Stress Waves in Solids (Dover, New York, 1963).

${ }^{18}$ R. J. Urick, Principles of Underwater Sound (McGraw-Hill, New York, 1975), 2nd ed.

${ }^{19}$ W. G. Neubauer, R. H. Vogt, and L. R. Dragonette, "Acoustic reflection from elastic spheres. I. Steady-state signals," J. Acoust. Soc. Am. 55, 1123-1129 (1974).

${ }^{20} \mathrm{~K}$. G. Foote, "Refined determination of the rigidity modulus by echo sounder measurement," in 1981 Ultrasonics Symposium Proceedings, Chicago, Illinois, 14-16 October 1981 (IEEE, New York, 1981), pp. 900-903.

${ }^{21} \mathrm{~T}$. Hasegawa and $\mathrm{K}$. Yosioka, "Acoustic-radiation force on a solid elastic sphere," J. Acoust. Soc. Am. 46, 1139-1143 (1969).

${ }^{22}$ F. Dunn, A. J. Averbuch, and W. D. O'Brien, Jr., "A primary method for the determination of ultrasonic intensity with the elastic sphere radiometer," Acustica 38, 58-61 (1977).

${ }^{23} \mathrm{~L}$. W. Anson and R. C. Chivers, "Frequency dependence of the acoustic radiation force function $\left(Y_{p}\right)$ for spherical targets for a wide range of mate- rials," J. Acoust. Soc. Am. 69, 1618-1623 (1981).

${ }^{24}$ R. C. Chivers and L. W. Anson, "Calculations of the backscattering and radiation force functions of spherical targets for use in ultrasonic beam assessment," Ultrasonics 20, 25-34 (1982).

${ }^{25}$ R. H. Vogt, L. Flax, L. R. Dragonette, and W. G. Neubauer, "Monostatic reflection of a plane wave from an absorbing sphere," J. Acoust. Soc. Am. 57, 558-561 (1975).

${ }^{26}$ L. Flax, L. R. Dragonette, and H. Überall, "Theory of elastic resonance excitation by sound scattering," J. Acoust. Soc. Am. 63, 723-731 (1978).

${ }^{27} \mathrm{D}$. N. MacLennan, "The theory of solid spheres as sonar calibration targets," Scottish Fisheries Research Report (22), pp. 1-17 (1981).

${ }^{28} \mathbf{K}$. G. Foote, "Constancy in target strength of optimal, elastic calibration spheres," in Proceedings of the IOA Underwater Acoustics Group Conference "Advances in Undenwater Acoustics," Portland, England, 1-2 December 1981 (Institute of Acoustics, Edinburgh, 1981), pp. 11/1-6.

${ }^{29} \mathrm{~K}$. G. Foote, H. P. Knudsen, and G. Vestnes, "Standard calibration of echo sounders and integrators with optimal copper spheres," Symposium of Fisheries Acoustics, Contribution No. 40, Bergen, Norway, 21-24 June 1982. 